\title{
The Effect of Measurement Limitations on High-Frequency Radar-Derived Spectral Energy Fluxes
}

\author{
Jean Clary, Louis-Philippe Nadeau, and CÉdric Chavanne \\ Institut des Sciences de la Mer de Rimouski, Université du Québec à Rimouski, Rimouski, Québec, Canada
}

(Manuscript received 20 December 2018, in final form 19 August 2019)

\begin{abstract}
The ocean's inverse cascade of energy from small to large scales has been confirmed from satellite altimetry for scales larger than $100 \mathrm{~km}$. However, measurements of the direct energy cascade to smaller scales have remained difficult to obtain. Here, the possibility of estimating these energy transfers to smaller scales from observations by high-frequency radars is investigated using numerical simulations. Synthetic measurements are first extracted from a quasigeostrophic simulation of freely decaying turbulence for which the reference energy flux is characterized by the transition from positive to negative values. Fluxes obtained from synthetic data are compared to this reference flux in order to assess the robustness to various measurement limitations. The geometry of the observational domain (nonperiodicity, domain size, and aspect ratio) affects mostly large scales, while the spatial resolution of the instruments affects mostly small scales. In contrast, measurement noise and missing data affect both large and small scales. Despite resulting in significant biases in the amplitude of the fluxes, the transition scale between the positive and negative fluxes is relatively robust to measurement limitations. These results are also confirmed using a simulation from a primitive-equations model in a realistic coastal geometry.
\end{abstract}

\section{Introduction}

The bulk of the energy input in the ocean is injected at large scales $(L>1000 \mathrm{~km})$ by lunisolar tides and atmospheric fluxes of momentum and buoyancy. In contrast, energy dissipation occurs at small scales $(L<1 \mathrm{~mm})$. Major gaps still exist in our understanding of how the ocean circulation closes this energy budget. In recent decades, it became clear that the dynamics of scales larger than the deformation scale are fundamentally different from those of the smaller scales.

At large scales, Earth's rotation constrains horizontal motions to be approximately in geostrophic balance (hereafter balanced motions). For relatively weak background potential vorticity (PV) gradient, these balanced motions are characterized by an inverse kinetic energy (KE) cascade (Charney 1971; Salmon 1980, 1998), for which energy is transferred from smaller to larger scales. Direct transfer of KE from larger to smaller scales can also occur locally in regions of strong background PV gradients, such as in western boundary currents (Straub and Nadiga 2014). Nevertheless, balanced dynamics

\footnotetext{
Corresponding author: Cédric Chavanne, cedric_chavanne@ uqar.ca
}

require parameterizations of motions smaller than the deformation scale in order to close the energy budget.

Earth's rotation plays a lesser relative role for scales smaller than the deformation radius, allowing dynamics to depart far from geostrophic balance (hereafter unbalanced motions). These unbalanced motions are generally characterized by a direct KE cascade from larger to smaller scales, en route to dissipation (McWilliams et al. 2001; Molemaker et al. 2005; Klein et al. 2008; Molemaker et al. 2010). Understanding how unbalanced motions are generated and interact with balanced motions is crucial to parameterize their role in closing the energy budget.

While significant progress has been achieved on understanding these mechanisms from a numerical standpoint (e.g., Vanneste and Yavneh 2004; Molemaker et al. 2005, 2010; Scott et al. 2011; Vanneste 2013; Nikurashin et al. 2013; Xie and Vanneste 2015; Taylor and Straub 2016), observations have remained scarce due to the high spatial and temporal resolution required. From an observational standpoint, most of our understanding of the ocean KE budget is derived from altimetry data (Scott and Wang 2005; Arbic et al. 2013, 2014), which has confirmed the existence of an inverse $\mathrm{KE}$ cascade of balanced motions at scales larger than 
$100 \mathrm{~km}$. However, exploration of the unbalanced smallscale motions from altimetry alone is not possible with the present-day spatial and temporal resolution of the satellite sensors. In addition, even with the expected increase in spatial resolution of future satellite missions, it is unclear how the unbalanced motions project on the sea surface height.

Recently, attempts to gain insight into KE transfer at scales smaller than the deformation radius has been made using measurements of surface currents from GPS-tracked drifters (e.g., Poje et al. 2017; Mensa et al. 2018). Using classical Kolmogorov scaling arguments, they show observational evidence for a direct energy cascade at scales smaller than $1-10 \mathrm{~km}$ and an inverse cascade at larger scales. However, Pearson et al. (2019) pointed out that the transition scale obtained using surface drifters is underestimated due to statistical biases.

A direct estimation of KE fluxes has been recently attempted using coastal surface currents from highfrequency radars (HFR; Soh and Kim 2018). HFR have a spatial resolution of roughly $1-5 \mathrm{~km}$, with a range of about $100 \mathrm{~km}$ and a temporal resolution of about $1 \mathrm{~h}$, enabling to capture both balanced and unbalanced motions (e.g., Ardhuin et al. 2009; Kim 2010; Chavanne et al. 2010; Kim and Kosro 2013). Soh and Kim (2018) applied standard spectral methods to HFR currents to estimate energy fluxes for horizontal scales ranging from 2 to $25 \mathrm{~km}$ and observed a wide range of behaviors including inverse and direct cascades. While promising, interpreting the signification of these results in the context of the ocean's KE budget is made challenging due to the unknown effects of various sources of observational errors on the estimation of KE spectra and fluxes.

Here, we systematically investigate the effect of the main sources of errors on estimating KE spectral fluxes using HFR measurements. To constrain the errors, we use model data instead of observations in order to derive an "ideal" reference flux. Synthetic observations with various sources of errors are then generated from the model data. Fluxes estimated from these synthetic observations are compared to the reference to quantify the effect of each source of error. In section 2, we present the numerical model and its energy budget, from which we define the reference KE flux. Then, in section 3, we estimate the effect of various error sources on the "observed" KE flux by comparing it to the reference one. Implications for estimating KE fluxes from actual HFR data are discussed in section 4.

\section{Reference kinetic energy flux}

Most recent numerical studies (e.g., Capet et al. 2008a,b; Klein et al. 2008; Tulloch et al. 2011; Straub and Nadiga 2014;
Arbic et al. 2014) predict KE fluxes that share the same essential qualitative structures: (i) an inverse cascade at large scales, (ii) a direct cascade at small scales, and (iii) a transition scale that coincides roughly with the deformation radius. We seek a reference flux that displays this basic structure, but with minimum complexity of the underlying physical model. The simplest configuration that yields these essential features is the quasigeostrophic model (Vallis 2006). Although smallscale unbalanced motions are parameterized by subgrid scale dissipation in this model, a range of positive fluxes results from its dynamics (see below) and occurs at similar scales than more complex primitive-equations models (e.g., Klein et al. 2008; Capet et al. 2008b; Arbic et al. 2013).

The simulations are performed using a simplified version of the numerical model described in Nadeau and Straub (2009). In the configuration used here, the model solves the two-layer quasigeostrophic potential vorticity equation, under the rigid-lid approximation. The thicknesses of the upper and lower layers are $H_{1}=$ $1000 \mathrm{~m}$ and $H_{2}=5000 \mathrm{~m}$, respectively. To facilitate the Fourier analysis required to compute the reference flux, the model geometry is a doubly periodic domain of equal length and width, $L_{\text {domain }}=2000 \mathrm{~km}$. The horizontal resolution is $d x=1.95 \mathrm{~km}$. Biharmonic dissipation, with $A_{h}=15 \times 10^{6} \mathrm{~m}^{4} \mathrm{~s}^{-2}$, is applied in both layers (see appendix A). Bottom drag is set to zero. The Coriolis parameter is $f_{0}=10^{-4} \mathrm{~s}^{-1}$ and the Rossby deformation radius is defined as $R_{d}=\sqrt{g^{\prime} H_{1} H_{2} / H f_{0}^{2}}$, where $g^{\prime}$ is the reduced gravity and $H=H_{1}+H_{2}$ is the total depth, and $R_{d}=30 \mathrm{~km}$.

The reference simulation is a standard freely decaying turbulence experiment (as in, e.g., Held et al. 1995; Scott 2001; Arbic and Flierl 2003). In addition to its simplicity, this configuration allows for spatially homogeneous turbulence statistics, which is required by our analysis of the effect of measurement errors on observed KE fluxes (see section 3a). The model is initialized using a baroclinically unstable vertical shear, sinusoidal in the meridional direction. Small random perturbations with wavenumbers $K=8 K_{1}$ to $K=$ $10 K_{1}$, where $K_{1}=2 \pi / L_{\text {domain }}$, are also added to this background shear in order to accelerate baroclinic instability.

Figure 1a shows a time series of KE in each layer, available potential energy (APE), and total energy in the reference simulation. The onset of baroclinic instability occurs at about 250 days, after which APE is progressively converted into KE. This transfer continues for a period of roughly 350 days, from which we arbitrarily select a time period to perform the analysis. Although fluxes depend on the specific choice of time 
a)
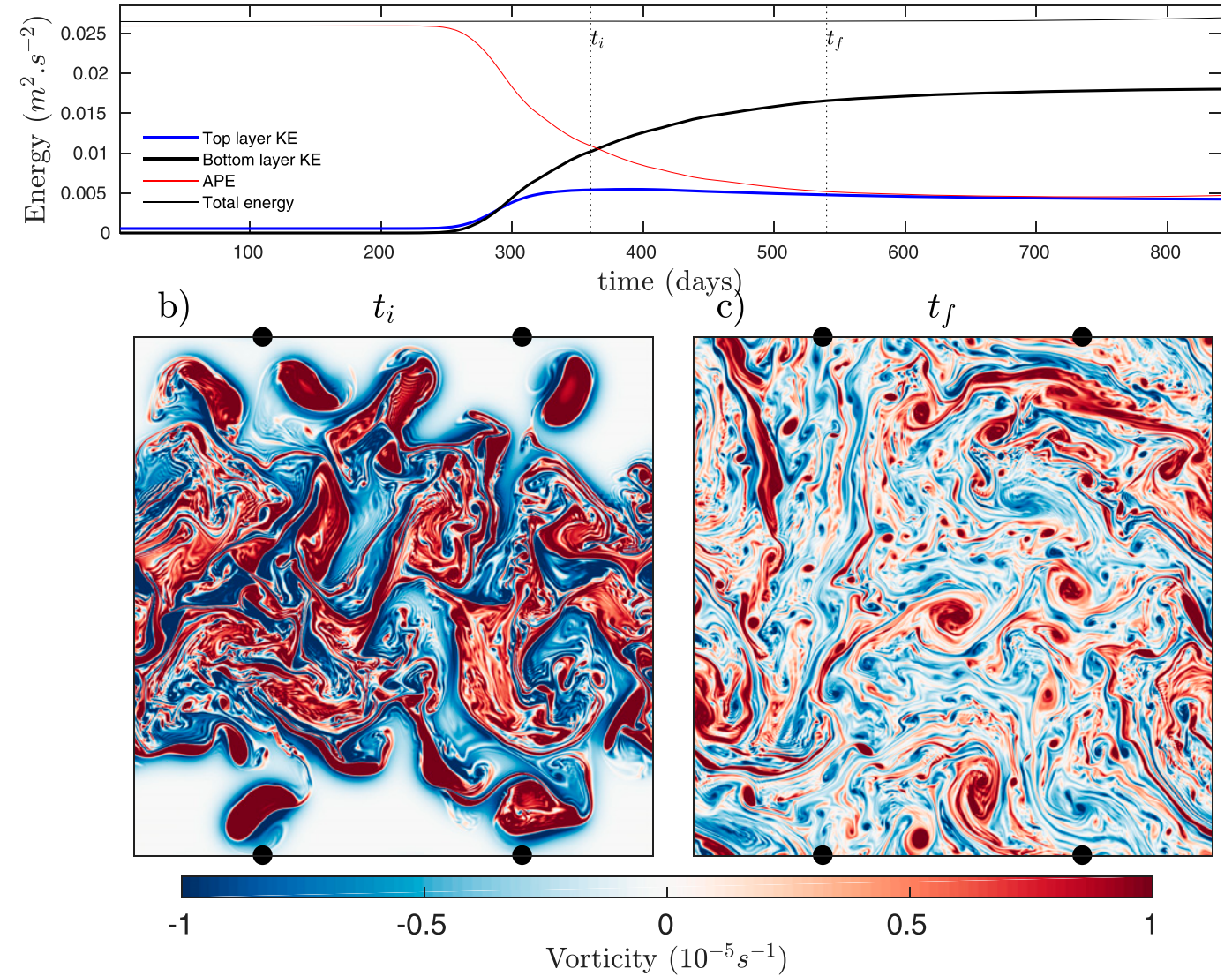

FIG. 1. (a) Times series of upper-layer kinetic energy (blue), bottom-layer kinetic energy (thick black), available potential energy (red), and total energy (thin black). Upper-layer relative vorticity at (b) $t_{i}$ and (c) $t_{f}$. Black dots represent synthetic radar positions.

period, results from the error analysis of next sections are independent from this choice as long as the time period is fixed (not shown). Examples of snapshots of upper layer relative vorticity at the beginning and end of this time period are shown in Figs. $1 \mathrm{~b}$ and 1c, respectively.

To describe the depth-averaged energy budget at a given spatial scale, we first express this budget in Fourier components

$$
-\frac{\partial}{\partial t}(\widehat{\mathrm{KE}}+\widehat{\mathrm{APE}})+\widehat{\Pi_{\mathrm{KE}}}+\widehat{\Pi_{\mathrm{APE}}}+\hat{D}=0,
$$

where $\widehat{\mathrm{KE}}$ and $\widehat{\mathrm{APE}}$ are Fourier spectra, $\widehat{\Pi_{\mathrm{KE}}}$ and $\widehat{\Pi_{\mathrm{APE}}}$ are transfer functions and $\hat{D}$ is the parameterized biharmonic dissipation. Each term of the energy budget [Eq. (1)] is detailed in Table A1 in appendix A. How the depth-averaged KE spectra evolve during the considered time period is shown in Fig. 2a. Each spectrum displays the typical -3 slope of geostrophic turbulence in the direct enstrophy cascade regime (Charney 1971).
$\widehat{\mathrm{KE}}$ increases at the largest and smallest scales, while decreasing at all other scales, indicating the presence of both an inverse and a direct energy transfer. To explain this behavior in more detail, we integrate each term of the energy budget [Eq. (1)] over time $t$ and wavenumber $K$ as follows:

$$
F(K)=\frac{1}{\Delta t} \int_{t_{i}}^{t_{f}} \int_{K}^{K_{\max }} \hat{F}\left(K^{\prime}, t^{\prime}\right) d K^{\prime} d t^{\prime}
$$

where $\Delta t=t_{f}-t_{i}$ and $\hat{F}$ represents a term in Eq. (1). This yields an integrated spectral energy budget

$$
\frac{-1}{\Delta t}(\Delta \mathrm{KE}+\Delta \mathrm{APE})+\Pi_{\mathrm{KE}}+\Pi_{\mathrm{APE}}+D=0,
$$

where $\Delta$ indicates differences between $t_{f}$ and $t_{i}, \Pi$ indicates energy fluxes, and $D$ is the energy sink due to dissipation. Each term of this integrated spectral energy budget is shown in Fig. 2b, along with their sum (error), which shows that the budget [Eq. (3)] is closed 
a) KE spectra

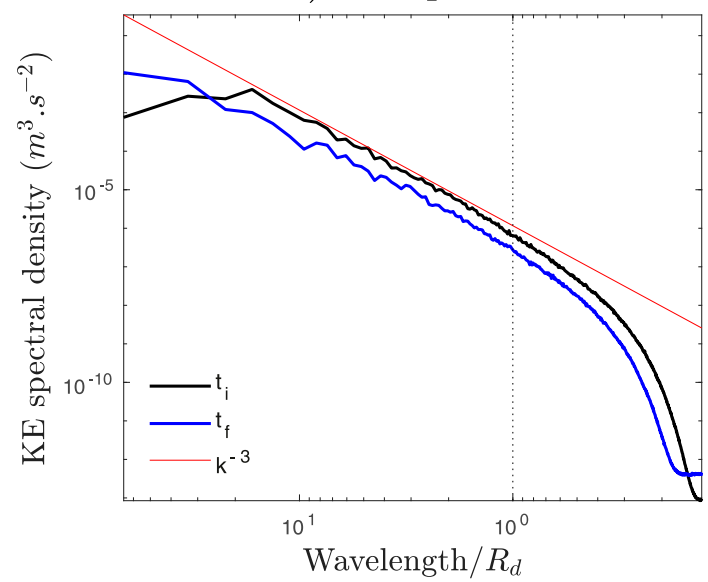

b) Energy budget

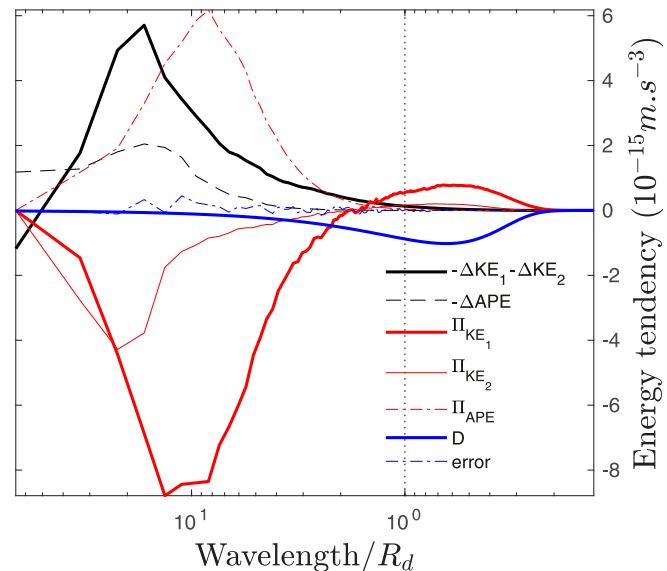

FIG. 2. (a) Upper-layer KE spectra at time $t_{i}$ (black) and $t_{f}$ (blue). (b) Spectral integral energy budget [Eq. (3)]. Terms in (b) are multiplied by the wavenumber such that the area under the curves represents the variance.

to a good accuracy. Notice that each term is multiplied by $K$, such that the area under the curves in Fig. $2 b$ represents the variance. ${ }^{1}$ To present fluxes in units of $\mathrm{m}^{2} \mathrm{~s}^{-3}$ and facilitate comparisons with other studies, the fluxes are displayed as $\left(R_{d} K H / H_{1}\right) \Pi_{\mathrm{KE}_{1}}$ in subsequent figures.

The interpretation of each term in Fig. $2 b$ depends on its role in the energy budget. For the difference terms $-\Delta$ the interpretation is straightforward: at a given scale $K$, a positive value indicates that the energy integrated over smaller scales decreased over $\Delta t$ (and vice versa for negative values). For the fluxes $\Pi$, a positive value indicates a transfer from larger to smaller scales (and vice versa for negative values). For both the difference and the transfer terms, local energy evolution at a certain scale $K$ is given by the slope of the curve. A positive slope indicates an increase of energy for the difference terms, but a decrease of energy for the transfer terms (and vice versa for a negative slope).

We choose $\Pi_{\mathrm{KE}_{1}}$ (Fig. 2b) as our reference flux, as it shares the same essential qualitative structures as those obtained with more complex Primitive-equations (PE) models: (i) a range of negative values is observed at large scales, (ii) a range of positive values is observed at small scales, and (iii) a transition occurs around $\lambda=2 R_{d}$. Notice that in this model, the range of positive values results from the parameterization of subgrid-scale dissipation, as $\Pi_{\mathrm{KE}_{1}}$ and $D$ almost balance each other at small scales.

\footnotetext{
${ }^{1}$ In a log-linear plot, the area under a curve is conserved when multiplying by the abscissa variable.
}

\section{Effects of measurement limitations}

We now systematically investigate conditions in which KE spectral fluxes can be estimated from HFRs measurements. Intuitively, we can expect that errors at large scales will arise from the geometry of the domain (limited extent, aspect ratio, nonperiodicity, etc.), while errors at small scales arise from measurement limitations (resolution, noise, missing data, etc.). Together, these two limits will define the range of scales over which KE fluxes can be estimated.

\section{a. Effects of domain geometry}

For oceanic observations, which are nonperiodic, estimating Fourier spectra requires the application of a window in order to reduce spectral leakage (Harris 1978). It is also common to remove the linear trend in order to reduce spurious energy at the largest scale (Emery and Thomson 2001). The effect of windowing on nonperiodic subdomains of arbitrary size $30 R_{d}$ is shown in Fig. 3a. To compare to the reference, we average fluxes obtained from $50 \%$ overlapping subdomains that cover the entire model domain. For nonperiodic subdomains, both large and small scales are affected if no window is applied (red curve). Applying a Hamming or a Hanning window reduces the errors at both large and small scales, but errors remain at small scales with the Hamming window, since periodicity is not fully enforced at the boundaries. ${ }^{2}$ We further tested other windows such as Blackman and Kaiser-Bessel and confirm that they

\footnotetext{
${ }^{2}$ Hamming coefficients do not tend to zero at the boundary.
} 

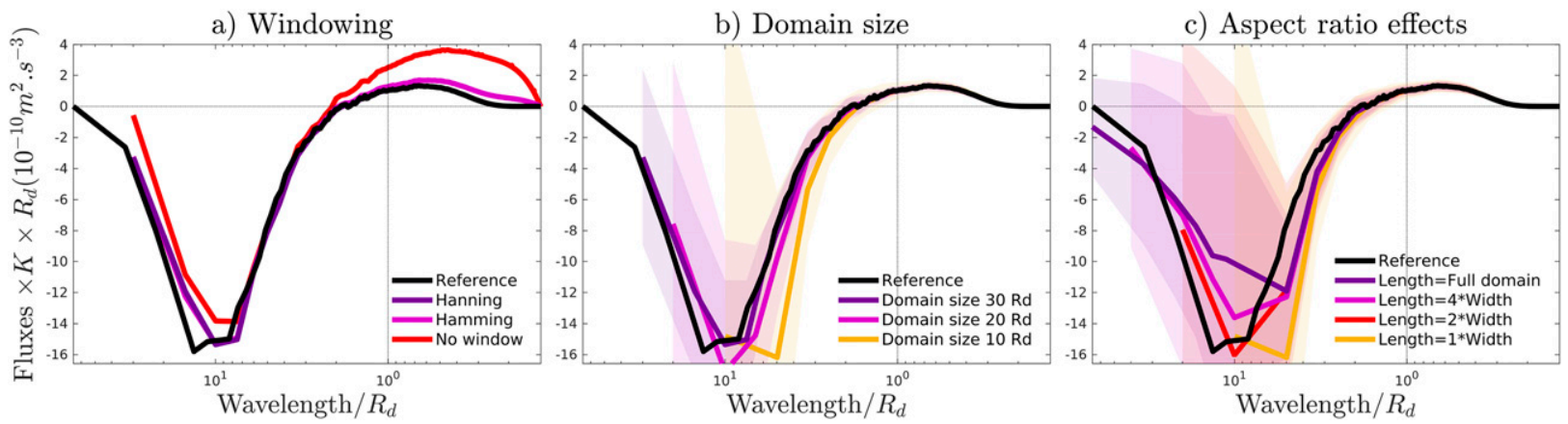

FIG. 3. Effect of the domain geometry on the spectral energy fluxes. (a) Windowing effect for nonperiodic subdomains of size $30 R_{d}$. (b) Effect of the size of square subdomains. (c) Effect of anisotropy for rectangular subdomains. In each panel, the reference flux of Fig. 2b is shown in black, and fluxes are multiplied by $R_{d} K$. Shaded areas show the standard deviations.

yield similar results to Hanning, which will be used hereinafter in the study.

The effect of the subdomain size from $30 R_{d}$ to $10 R_{d}$ is shown in Fig. 3b. For all subdomain sizes, errors at small scales remain negligible. However, errors at large scales increase as the horizontal extent decreases. In particular, for $10 R_{d}$, the peak of negative flux is shifted to smaller scales, without however affecting the transition scale.

Above, we have only considered squared subdomains, for which the integration in Fourier space is isotropic. How the anisotropy introduced by a rectangular subdomain affects the spectral fluxes is shown in Fig. 3c, for a fixed width of $10 R_{d}$. Increasing the aspect ratio of the subdomain decreases the magnitude of the negative peak. Paradoxically, since the latter is overestimated for a squared subdomain of $10 R_{d}$, increasing the aspect ratio reduces the error initially for a ratio of 2 (red curve) but increases the error for larger ratios.

\section{b. Synthetic radar measurements}

So far, we estimated spectral fluxes directly from the model data. We now investigate the effects of the various measurements limitations by mimicking radar data from the numerical model. To simulate the effect of the radar measurement, two fundamental steps must be followed: (i) for each radar, model currents must be projected onto the radial axes of a polar grid (hereafter "current measurement"), and (ii) radial currents from multiple radars must be mapped onto a common Cartesian grid (hereafter "current mapping"). This yields the two components of the surface currents, $\mathbf{u}_{h}=(u, v)$, from which we can compute the spectral fluxes as follows:

$$
\Pi(K)=-\frac{1}{\Delta t} \int_{t_{i}}^{t_{f}} \int_{K}^{K_{\max }} \operatorname{Re}\left[\widehat{\mathbf{u}}_{h}^{*} \cdot \overline{\mathbf{u}_{h} \cdot \nabla_{h} \mathbf{u}_{h}}\right] d K^{\prime} d t^{\prime},
$$

where $\nabla_{h}=\left(\partial_{x}, \partial_{y}\right)$ is the horizontal $2 \mathrm{D}$ gradient operator.
To disentangle the effects of the radar measurement from those of the domain geometry, we begin our investigation from an ideal but unrealistic configuration, which has the following characteristics:

- Four HFRs are monitoring the entire domain, with two on each of the south and north sides (black bullets in Fig. 1).

- The range and aperture of each HFR covers the entire domain.

- The radial resolution equals the numerical horizontal resolution of $2 \mathrm{~km}$.

- The angular resolution equals $d \theta=0.25^{\circ}$.

- HFR measurements have no noise or missing data.

In this ideal configuration, we assume that the measurements range is unlimited, allowing us to test the effect of current mapping on the doubly periodic domain. Since the aspect ratio of the radar grid cells increases with the range, this implies strong deformations away from the radar. To keep a reasonable aspect ratio at the furthest distance from each radar, we use an angular resolution $d \theta=d \theta_{r} L_{r} / L_{\text {domain }}$, where $L_{r}=$ $100 \mathrm{~km}$ and $d \theta_{r}=5^{\circ}$ are standard HFR range and angular resolution, respectively.

Subdomains from this ideal configuration will be later considered that are meant to represent HFR stations monitoring an estuary or a coastline with offshore islands. In this latter configuration, realistic range and angular resolutions will be used. Mapping currents from HFRs requires a minimum of two HFRs at different locations, but additional HFRs monitoring a given area can improve the current mapping accuracy.

Current measurement implies averaging as HFRs perform an averaging over range and azimuth when measuring surface velocities (Ohlmann et al. 2007). Averaging depends on the range and resolution of the radar. Here in practice, the Cartesian grid of the numerical model has a finite resolution and some of 
the radar grid cells near the radar do not contain any Cartesian grid point. For the area corresponding to those grid points, an interpolation is needed, while an averaging is needed over the rest of the domain. To go around this issue, we first interpolate by nearest neighbor each component of the surface velocity onto a highazimuthal-resolution polar grid centered at the HFR. The azimuthal resolution is chosen such that the grid cells farthest away from the radar have a size matching that of the Cartesian grid cells. These high-resolution data are then averaged onto the radar grid with a simple azimuthal boxcar filter. To obtain the current measurement, the averaged velocity is projected into the direction of the radar. Finally, all radar measurements are mapped onto a Cartesian grid of resolution equal to the radar's radial resolution.

\section{c. Effects of current mapping}

Several methods are available to perform current mapping of the radar measurements. Figure 4 shows the effect of four standard methods on the current mapping accuracy. The methods, displayed in Figs. 4a-d, respectively, are unweighted least squares (UWLS; Lipa and Barrick 1983), weighted least squares (WLS; Kim et al. 2008), optimal interpolation (OI; Kim et al. 2008), and variational interpolation (2Dvar; Yaremchuk and Sentchev 2009). Of all the tested methods, the variational interpolation method yields the smallest errors. This method requires, however, a carefully set number of smoothing parameters (see appendix B). In contrast, all the other methods tested only require the setting of a single parameter (corresponding to a decorrelation scale) but yield larger errors. Among them, the OI method performs almost as well as 2Dvar, offering a good compromise between accuracy and robustness to the choice of parameters.

Figure 5a shows the effect of using each of the four mapping methods on the spectral fluxes computed from Eq. (4). As expected from the vorticity errors shown in Fig. 4, both least squares methods introduce significant biases in the spectral fluxes. The variational method recovers almost perfectly the reference flux, followed closely by the OI method. In the following, we elect variational interpolation as the mapping method when considering current measurement errors.

\section{d. Effects of radar resolution}

We investigate the influence of measurement resolution on the energy fluxes by varying independently the radial resolution (tied with the Cartesian grid resolution) and the azimuthal resolution (Fig. 5b). As expected, the effect of resolution has very limited impact on the large scales, but induces biases at small scales. In particular, decreasing the resolution shifts the transition from positive to negative fluxes to larger scales [similar to an averaging performed in Arbic et al. (2013)]. This effect is observed both for decreasing the radial resolution and the azimuthal resolution independently. Larger biases are observed for combined changes in resolution.

\section{e. Effects of measurement noise}

Following Forget (2015), we mimic measurement noise by adding white noise to the radial currents obtained in our ideal configuration, $U_{\text {ideal }}: U_{\text {obs }}=U_{\text {ideal }}+\nu V w$, where $V$ is the root-mean-square magnitude of the current, $w$ is a random number between -1 and +1 taken from a uniform distribution, and $\nu$ sets the signal-to-noise ratio (SNR) of $3 \nu^{-2}$.

Examples of vorticity fields for different noise levels are shown in Fig. 6. Noise mostly affects small scales, but its effect on energy fluxes spreads to large scales as well (Fig. 5c). Specifically, adding noise has only a limited impact on the transition scale, but affects the amplitude of the two main lobes. In particular, the amplitude of the negative lobe is strongly decreased for a noise level above $20 \%$.

\section{f. Effects of missing measurements}

HFR measurements are not only limited by resolution and noise, but also by gaps and by radar shutdowns, which can strongly affect the current mapping accuracy. In general, gaps in radar coverage are caused by electromagnetic interferences, by low signal-to-noise ratios, and by the type of algorithm used to retrieve the signal direction (beam-forming or direction-finding).

To account for realistic gap distributions, spatial structures originating from actual radar measurements have been imposed on the synthetic radar data. An example is shown in Fig. 7a. Different gap distributions, such as the one shown in Fig. 7a, are then randomly selected for each of the four HFRs. Figure $7 \mathrm{~b}$ shows an example of radar coverage where each Cartesian grid point is assigned a number ranging from 0 to 4 , depending on the number of radial currents measurements from different HFRs falling in the Cartesian grid cell. To quantify the amount of missing data, we define a percentage of the total area covered by the radars, $P$. Since at least two independent measurements are necessary to map the currents at a given grid point, this percentage is defined as follows: $P=$ $1-\left(2 N_{0}+N_{1}\right) /(2 M)$, where $N_{0}$ is the number of grid points with zero measurement, $N_{1}$ is the number of grid points with only one measurement and $M$ is the total number of grid points. ${ }^{3}$ For the example in Fig. $7 b$, the data coverage is $P=0.746$.

\footnotetext{
${ }^{3}$ Notice, however, that this definition is nonunique as many combinations of $N_{0}$ and $N_{1}$ can lead to the same value of $P$.
} 
a) UWLS

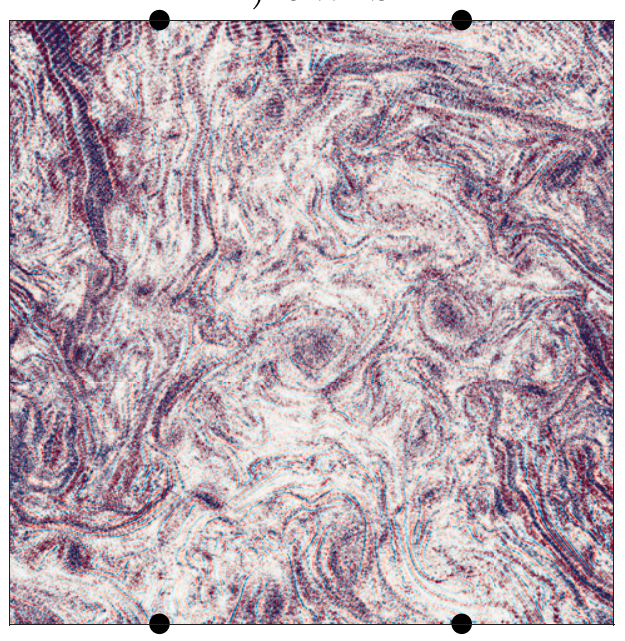

c) $\mathrm{OI}$

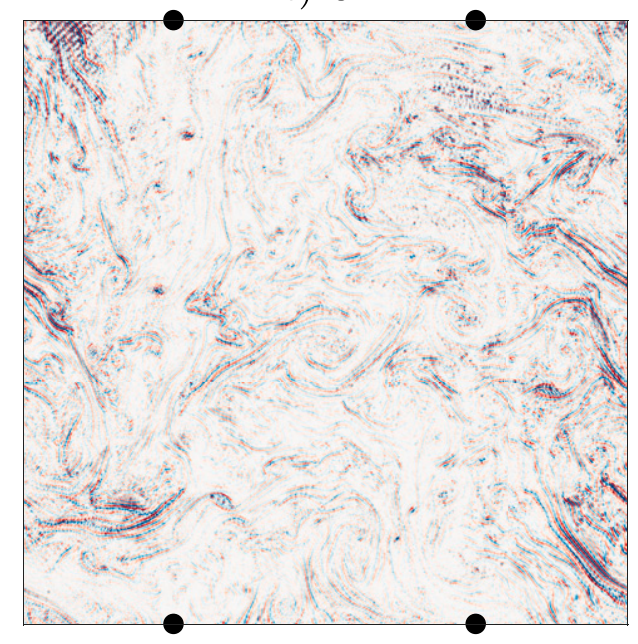

b) WLS

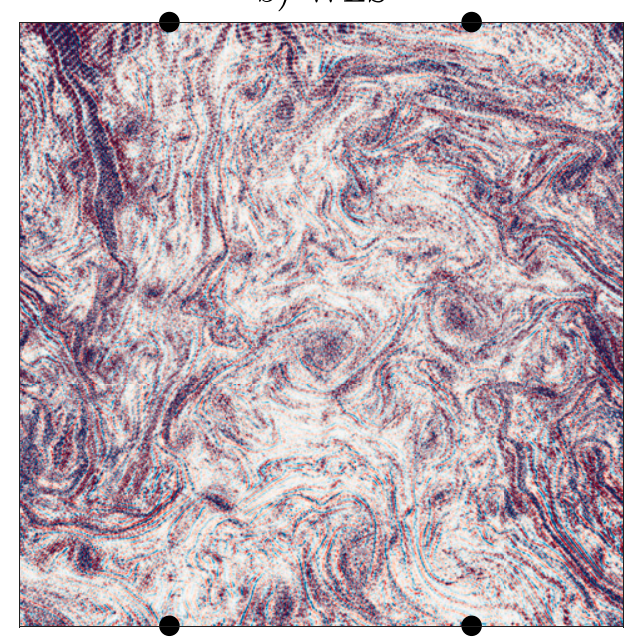

d) 2Dvar

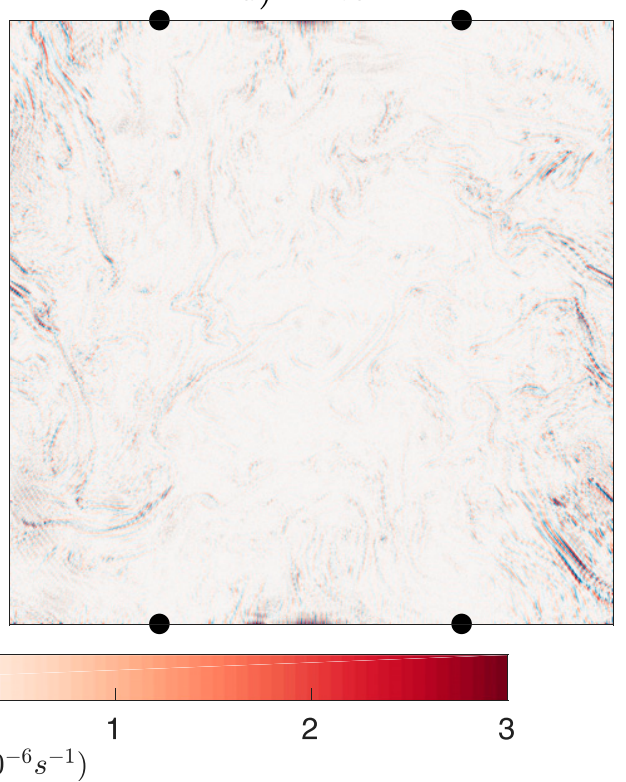

FIG. 4. Errors in relative vorticity $\left(\mathrm{s}^{-1}\right)$ for a snapshot sampled with different mapping methods: (a) unweighted least squares, (b) weighted least squares, (c) optimal interpolation, and (d) variational interpolation. Black dots represent synthetic radar positions.

The effect of varying the area covered by the radars on the spectral fluxes is shown in Fig. 5d. As for the measurement noise, the effect of gaps is limited essentially to the amplitude of the lobes, although the transition scale is less robust to missing data (for $P<70 \%$ ) than to noise.

\section{g. Effects of radar shutdowns}

In addition to spatial gaps, we also consider the case of radar shutdown. To separate both effects, we assume that the available radars have no gap, while data from one or two radars are completely missing. When only one radar is shut down, the currents are still well mapped from the measurements of the three other radars (Fig. 8a), with minimal impact on the KE flux (Fig. 5e).

When two radars are shut down, the two available radars are either 1) on the same side, 2) facing each other, or 3) diagonally aligned. These configurations have different impacts on the current mapping accuracy (Fig. 8). In particular, large errors occur where the angle between the two radial directions is close to $0^{\circ}$ or $180^{\circ}$, a phenomenon known as geometric dilution of precision (GDOP; Chapman et al. 1997). GDOP is clearly 
a) Mapping method

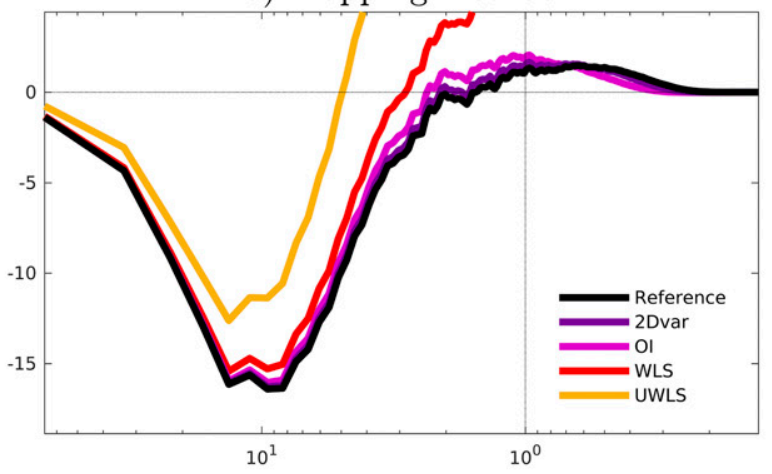

c) Noise

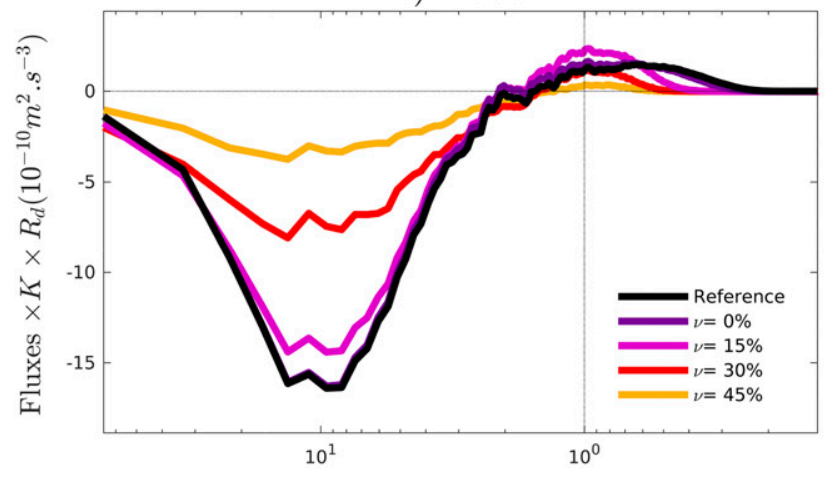

e) HFR shutdown

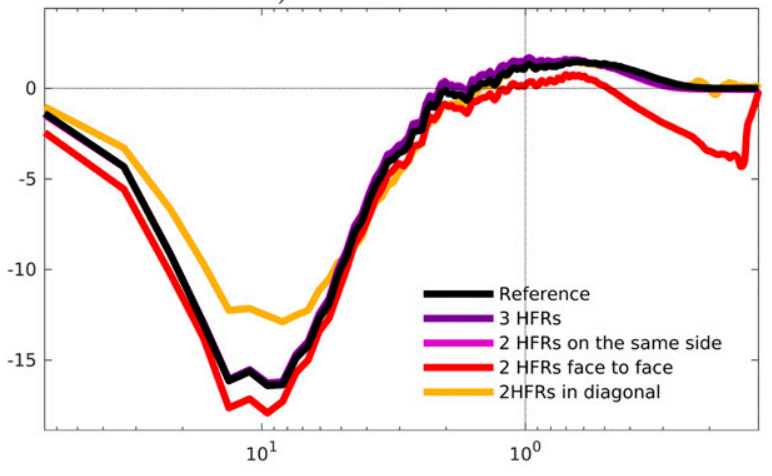

b) HFRs resolution

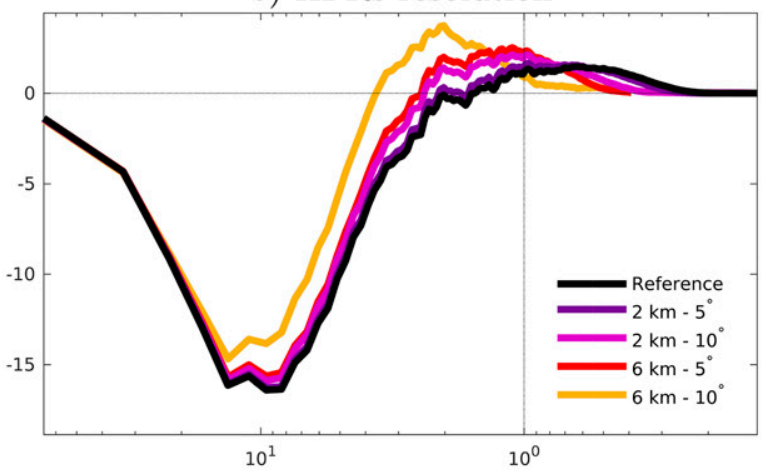

d) Missing data

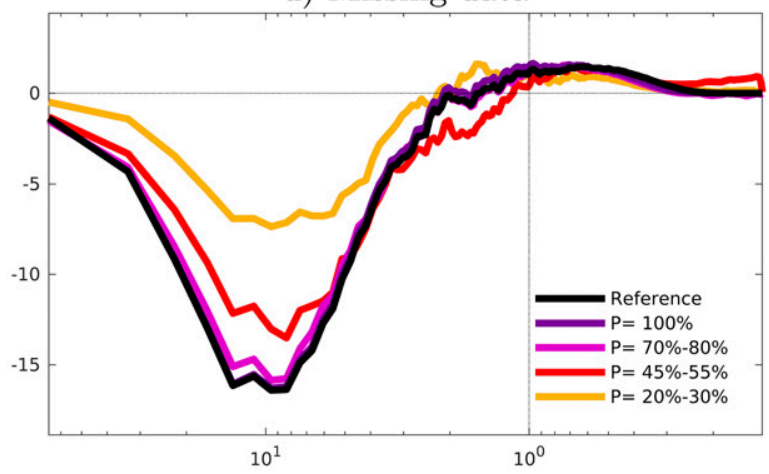

f) Combination of errors

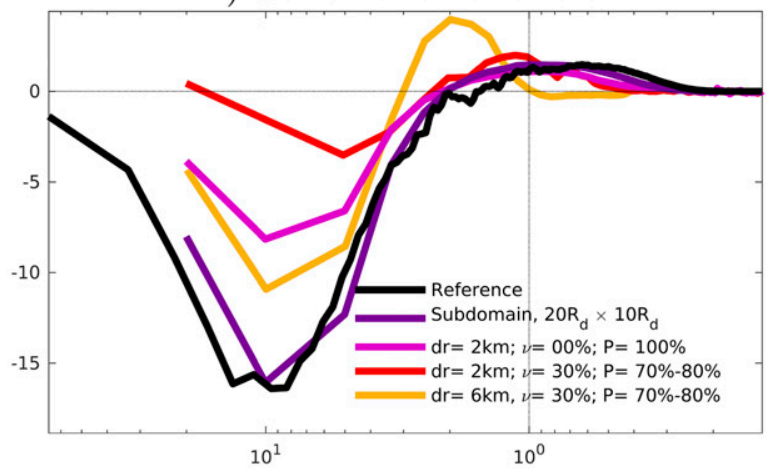

Wavelength $/ R_{d}$

FIG. 5. Effects of current measurement and mapping on the spectral energy fluxes. (a) Effect of current mapping in the ideal configuration (see text). The 2Dvar mapping method is used for all other panels. (b) Effects of radar resolution. (c) Effects of measurement noise using the ideal resolution. (d) Effects of missing measurements. (e) Effects of radar shutdowns. (f) Effects of measurement and mapping error combinations for subdomains of size $20 R_{d} \times 10 R_{d}$. In each panel, the reference flux (shown in black) is computed using a Hanning window and therefore differs slightly from that shown in Fig. 2b, and fluxes are multiplied by $R_{d} K$.

visible on the cases where radars are on opposite sides of the domain, where large errors appear along the line connecting the radars. When the radars are on the same side, GDOP effects are minimized on the line connecting the radars but amplified away from the radars (see Fig. 8b). The effects of GDOP on the KE flux are shown in Fig. 5e. As expected, strong biases exist in the cases where the radars are on opposite sides. Fluxes are better estimated for the case where radars are on the same side since the bulk of the mapping errors are reduced by windowing near the boundaries. Notice, however, that other limitations 
a) $\nu=0 \%$

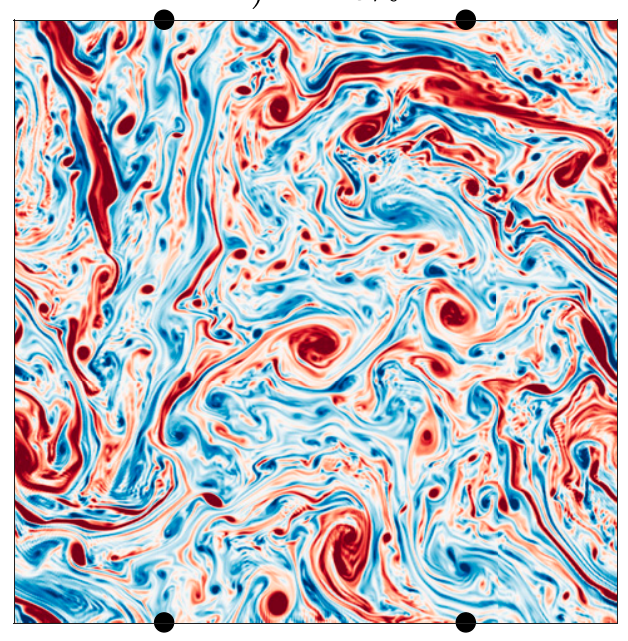

c) $\nu=30 \%$

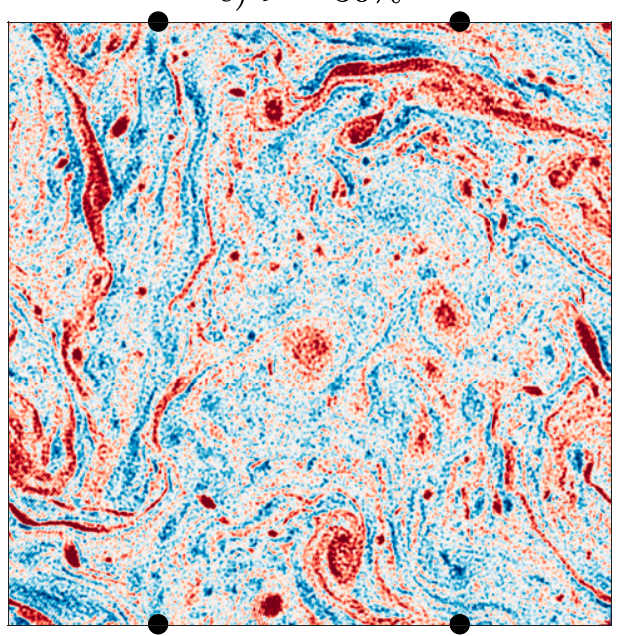

b) $\nu=15 \%$

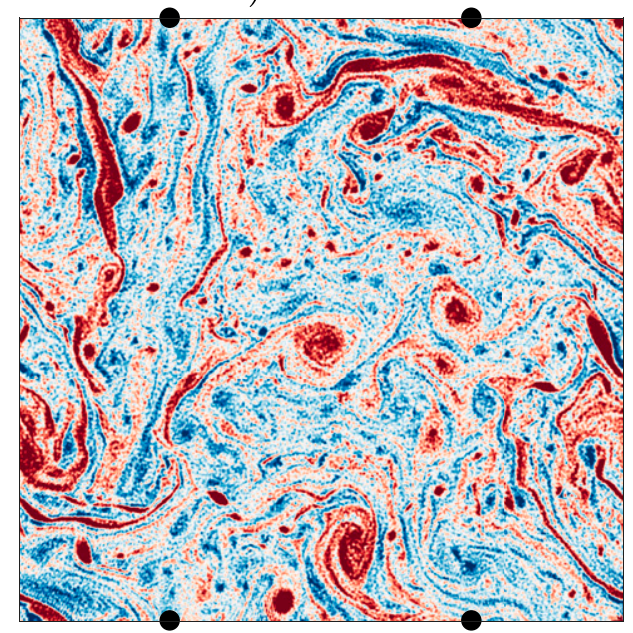

d) $\nu=45 \%$

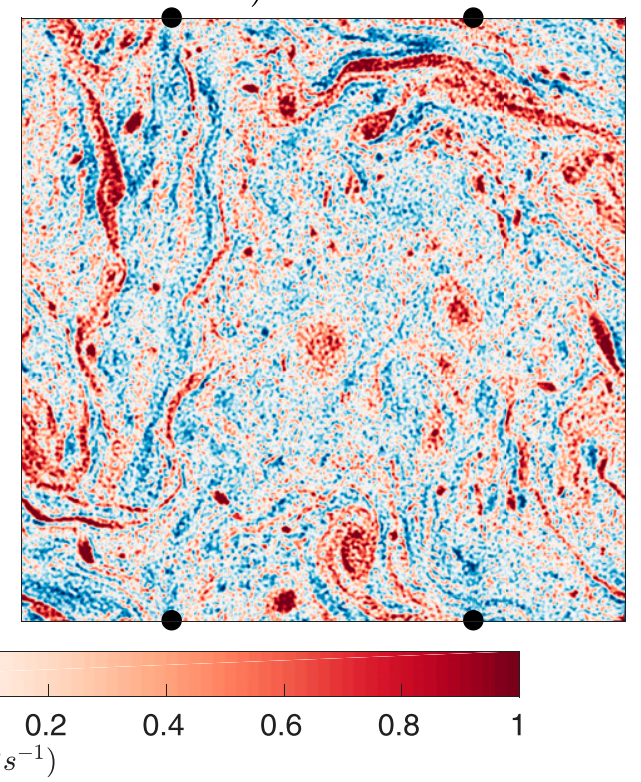

FIG. 6. Measurement noise effect on relative vorticity mapping for noise levels of (a) $\nu=0 \%$, (b) $\nu=15 \%$, (c) $\nu=$ $30 \%$, and (d) $\nu=45 \%$. Currents are mapped with the 2Dvar method.

such as missing data (resulting from, e.g., insufficient range) and noise are not taken into account here, and their effects may be exacerbated when only two radars are used.

\section{h. Combination of errors}

We now attempt to combine the above measurement limitations. Here we choose to consider only a limited set of combinations that are most likely to be relevant for the real HFR measurements. We first select a rectangular subdomain of $20 R_{d}$ in length by $10 R_{d}$ in width. The effect of this domain geometry alone (without any radar measurement effects) on the spectral fluxes is reproduced on Fig. $5 \mathrm{f}$ (purple curve). As shown previously, only the largest scales are affected by the domain geometry. The effect of current mapping of this subdomain using noise-free synthetic radar measurements is shown in pink. Here, biases are induced at both small and large scales, although the transition scale is still captured adequately. Introduction of $30 \%$ of measurement noise and $25 \%$ of missing data adds additional biases, but still captures the transition scale (red curve). Finally, degrading the radial resolution from 2 to $6 \mathrm{~km}$ shifts the transition scale to larger values and increases the amplitude of the positive lobe (yellow curve). 

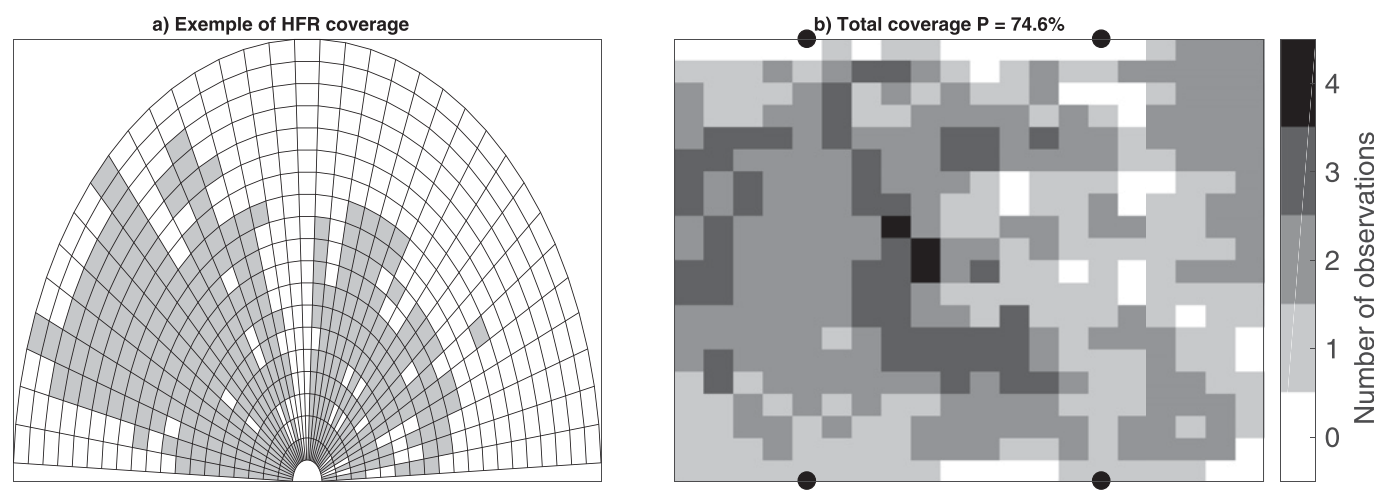

FIG. 7. Example of hourly data coverage from (a) an actual HFR (CODAR station in the St. Lawrence Estuary) and (b) four synthetic HFRs with random individual data coverage similar to that shown in (a).

\section{Discussion and conclusions}

One of the main appeals of using HFR data to compute spectral energy fluxes is to identify the limit between large-scale balanced dynamics and smaller-scale unbalanced motions. Here, we show that despite the introduction of significant biases in the general structure of the positive and negative lobes, the transition scale between these lobes is relatively robust to measurement limitations. The main effect comes from the HFRs resolution which shifts the transition scale to larger scales due to the implicit averaging by the radar sampling. Similar results were previously observed by Arbic et al. (2013) using a PE model to mimic the averaging performed when mapping satellite altimetric data. Nevertheless, in all cases considered in this study, this shift remained much smaller than an order of magnitude from the reference value. This suggests that the transition scale could be reasonably captured using HFR measurements.

However, our experimental design suffers from two main caveats that could modify the main conclusions of this study. First, the statistical properties of the upperlayer circulation considered in our freely decaying experiments do not undergo fast variations in time, in contrast to the real ocean circulation. In practice, this implies an upper limit on the time interval that can be used for averaging. Our results from the simple idealized configuration show that the transition scale quickly converges within a factor of 5 of the typical turbulent turnover time scale (not shown). This convergence could be significantly delayed or even nonexistent when considering oceanic data. The second important limitation of our configuration is the choice of a quasigeostrophic framework, which does not allow for an explicit representation of the small-scale unbalanced motions. As such, the effects of measurements errors could differ when a new type of dynamics is included.
To evaluate the effects of the above caveats, we briefly describe results from a primitive-equations model used in a regional configuration with realistic forcing. ${ }^{4}$ Figure 9a shows a snapshot of surface relative vorticity over the gulf and estuary of Saint Lawrence. Tidal frequencies from the surface velocity fields have been removed using harmonic analysis (Pawlowicz et al. 2002) to filter out the strong tidal components of the currents in the Saint Lawrence estuary. To compute spectral fluxes from the model data, we first select a subdomain in the lower Saint Lawrence estuary (Fig. 9b), where actual radar stations are operated (black dots; Kamli et al. 2016). We also focus on a time period corresponding to November 2015, for which the averaged stratification yields a first internal Rossby radius $R_{d} \approx$ $7 \mathrm{~km}$. Geostrophic currents are extracted from the filtered surface fields by computing the nondivergent component via Helmholtz decomposition. Figure 9c shows a timemean KE spectrum obtained using these geostrophic currents. For comparison, we also show the QG spectrum and normalize the wavenumbers of each model by their respective domain size. Both spectra yield roughly the same -3 slope. The reference flux obtained directly from the PE model geostrophic currents (red curve in Fig. 9c) shares the same qualitative structure as the one obtained from the QG model (black curve in Fig. 3a). The transition scale corresponds approximately to $\lambda=0.8 R_{d}$, which is 2.5 times smaller than the value obtained from the $\mathrm{QG}$ model. The effects of radar current mapping using radial

\footnotetext{
${ }^{4}$ The PE simulation originates from a nested high-resolution $(\sim 500 \mathrm{~m})$ version of the NEMO model covering the Gulf of Saint Lawrence, provided by Environment and Climate Change Canada at Dorval (F. Roy 2018, personal communication). The model is forced with open boundaries at the interface with the Atlantic and atmospheric forcing from the Global Environmental Model (GEM).
} 


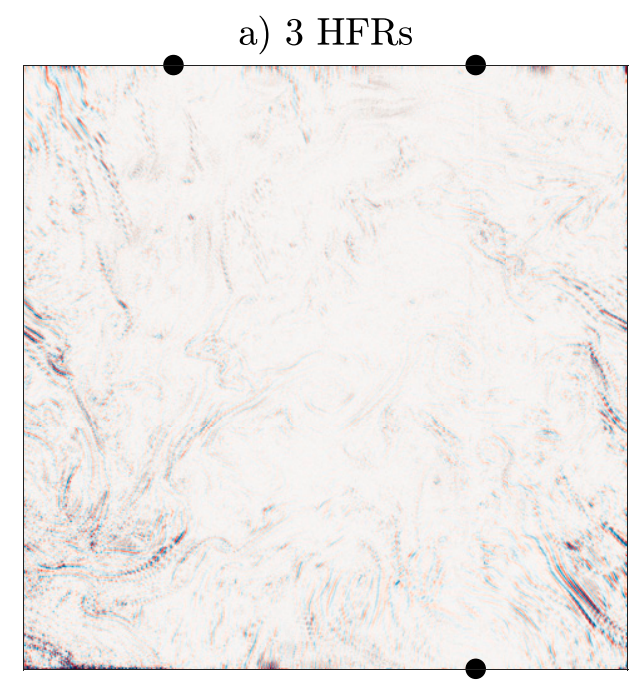

c) 2 HFRs face to face

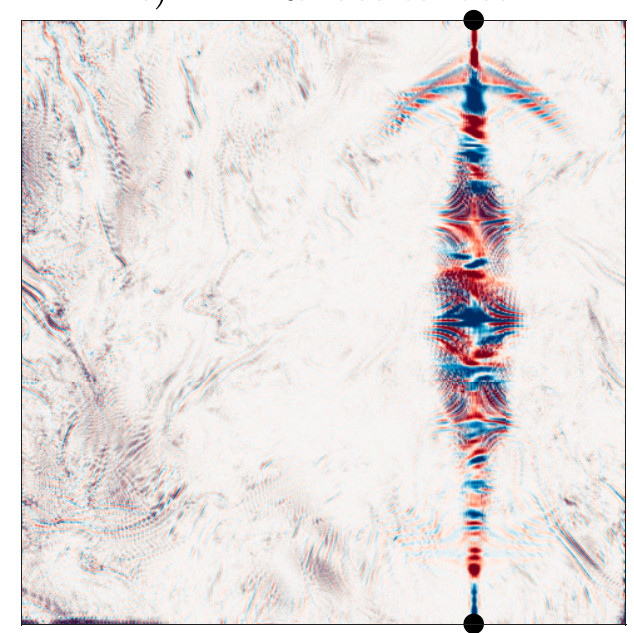

b) 2 HFRs on same face

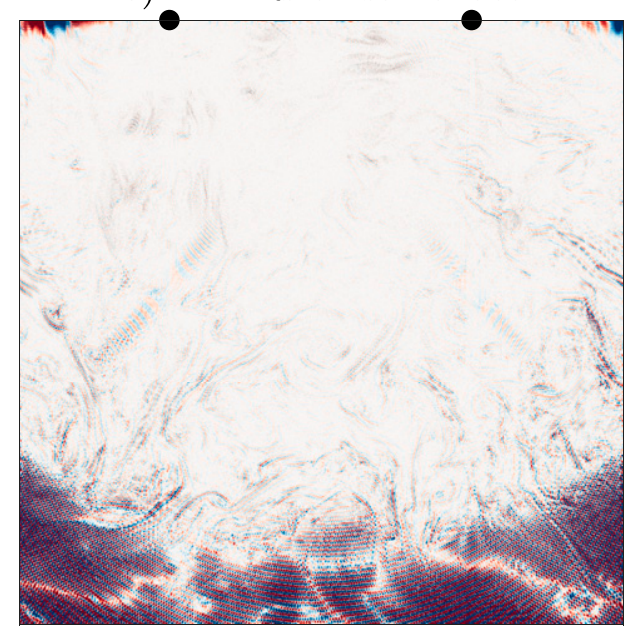

d) 2 HFRs in diagonal

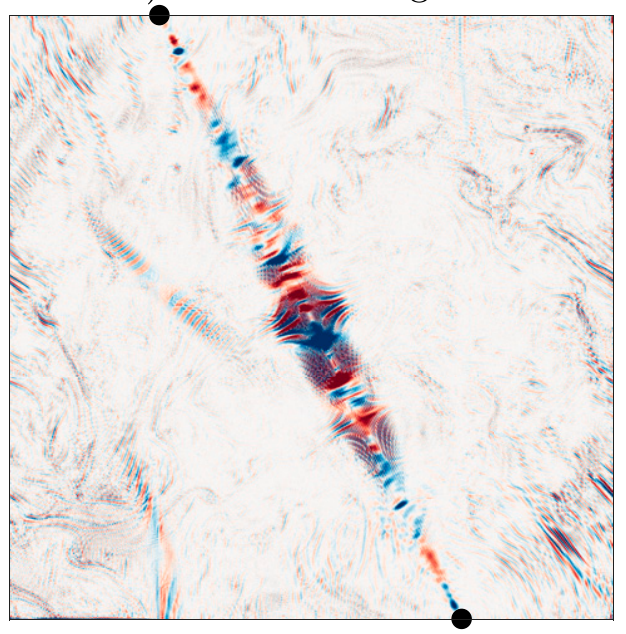

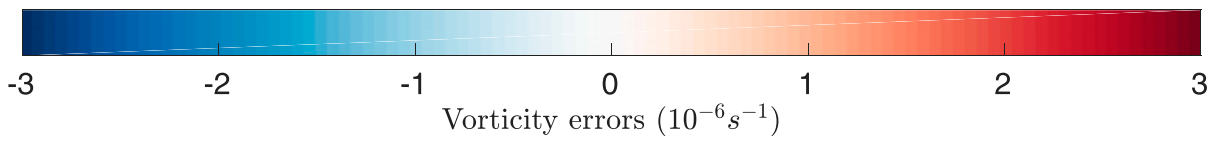

FIG. 8. Errors in relative vorticity for a snapshot sampled with (a) 3 HFRs and (b) 2 HFRs on the same side of the domain and (c),(d) 2 HFRs on opposite sides. Currents are mapped with the 2Dvar method.

and azimuthal resolutions corresponding roughly to those of the actual radars are shown in black and blue. The averaging performed by the radar sampling shifts the transition scale to larger values, consistent with the results obtained with the QG model (Fig. 5b). Contrary to what is shown in Fig. 9d, averaging also increases the positive lobe amplitude of KE fluxes, as shown in previous studies (e.g., Arbic et al. 2013). Recall that in order to conserve the area under the curve, the fluxes are multiplied by $K$, which magnifies the high wavenumbers.

The results shown in Fig. 9 should be viewed as a preliminary best case scenario, where only the effects of mapping and resolution are considered. A more thorough investigation including comparison with actual radar data will be presented in a subsequent study. Yet, these preliminary results suggest that it appears possible to estimate KE fluxes from HF radar measurements of surface currents over a limited range of scales. The largest scale is limited by the radars range, which can reach a few hundreds of kilometers at the lowest HF frequencies. The smallest scale is limited by the radars radial and azimuthal resolutions and by the noise level. Radial resolution can reach a few hundreds of meters at the highest HF frequencies. The observable scales therefore typically range from a 

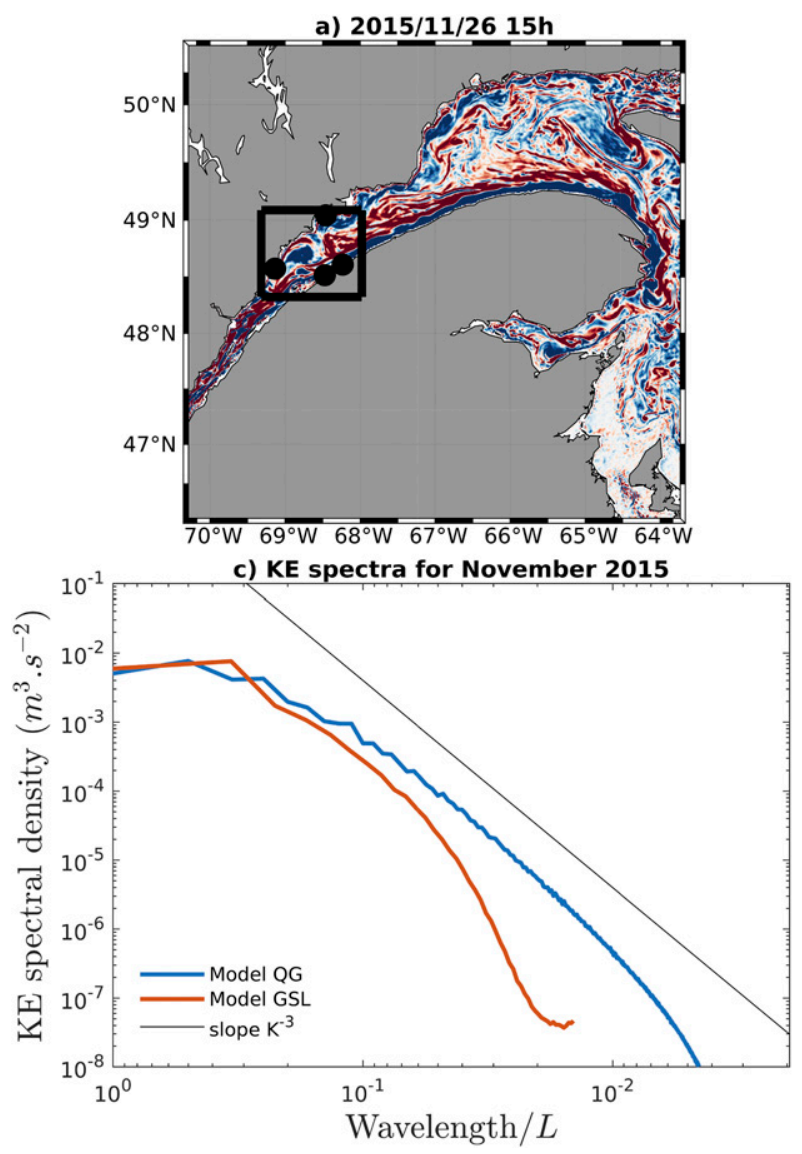

b) $2015 / 11 / 2615 \mathrm{~h}$

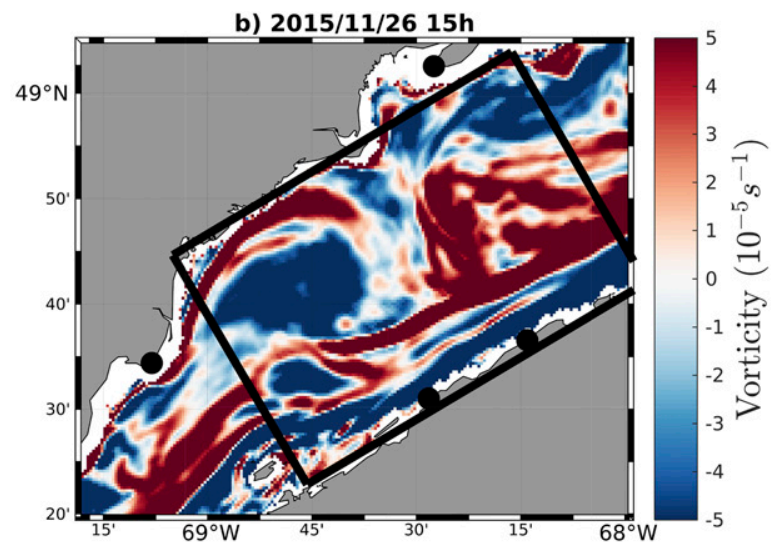

d) Fluxes for November 2015

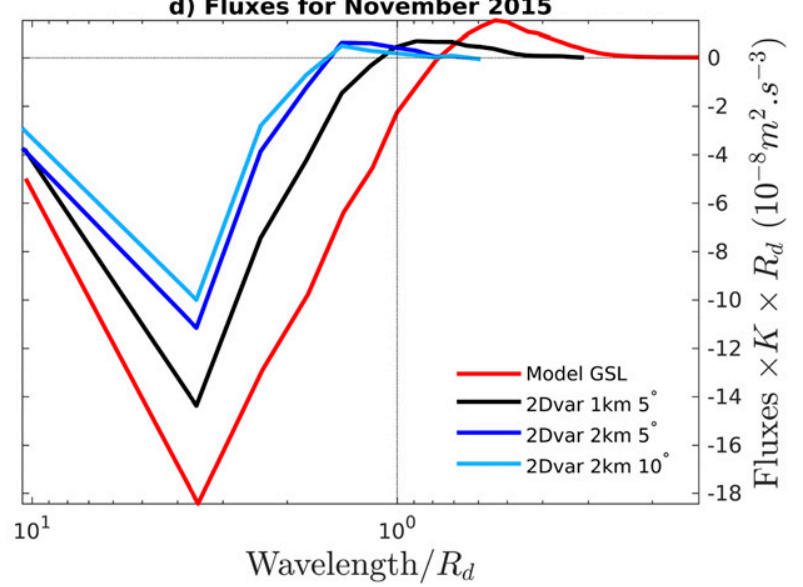

FIG. 9. Results from the primitive-equations model simulation in a regional configuration (GSL run). (a) Example of a snapshot of detided surface relative vorticity in the estuary and the Gulf of Saint Lawrence. Black dots show the positions where actual HFRs are operated. (b) Zoom-in of the black rectangle in (a). The black rectangle in (b) shows the area used for the analysis. (c) Time-averaged KE spectrum using the geostrophic currents from the GSL run for November 2015 (red). The spectrum is compared to that of the QG model at time $t_{f}$ (blue). Wavelengths are normalized by the respective model domain sizes. (d) Effect of radar resolution on the reference KE flux of the GSL run (red). Fluxes are obtained from noise-free synthetic HFR measurements at various radial and azimuthal resolutions (black and blue).

few kilometers to a few tens of kilometers, which fortunately corresponds to the transition scale between the direct and inverse KE cascade. However, measurements constraints introduce biases in the estimation of the positive and negative flux lobe amplitudes.

By allowing one to estimate the transition scale from negative to positive fluxes in coastal areas, HFRs could be a useful tool to gain information on the observed KE budget in a range of scales relevant for the small unbalanced motions. To extend this estimation to the open ocean would however require a different type of instrument such as a spaceborne high-resolution altimeter or a synthetic aperture radar. Yet, even in the best case scenario where their horizontal resolution allows for flux estimates well beyond the transition scale, these types of measurements will remain limited in their temporal resolution. We anticipate that hourly
HFR measurements could be used to interpret and validate spaceborne flux estimates.

Acknowledgments. Financial support to J. Clary was provided by NSERC (Grants 418247-2012-RGPIN and RGPIN-2018-06585 to Chavanne), ISMER, and Québec Océan. We thank François Roy and Simon Senneville for providing regional model data and James Caveen for his technical support. We also thank Alexei Sentchev and Max Yaremchuck for providing the 2Dvar code and Sung Yong Kim for providing the optimal interpolation code.

\section{APPENDIX A}

\section{Depth-Averaged Energy Budget}

The two-layer quasigeostrophic potential vorticity equations (Vallis 2006) are 
TABLE A1. Depth-averaged spectral energy terms.

\begin{tabular}{|c|c|}
\hline Name & Expression \\
\hline$\widehat{\mathrm{APE}}$ & $\begin{array}{c}\left(H_{1} \operatorname{Re}\left[{\widehat{\psi_{1}}}^{*} \widehat{\nabla}^{2} \psi_{1}\right]+H_{2} \operatorname{Re}\left[{\widehat{\psi_{2}}}^{*} \widehat{\nabla}^{2} \psi_{2}\right]\right) / H \\
-\frac{f_{0}^{2}}{g^{\prime} H} \operatorname{Re}\left[\left(\overline{\psi_{1}-\psi_{2}}\right)^{2} / 2\right]\end{array}$ \\
\hline$\widehat{\Pi_{\mathrm{APE}}}$ & 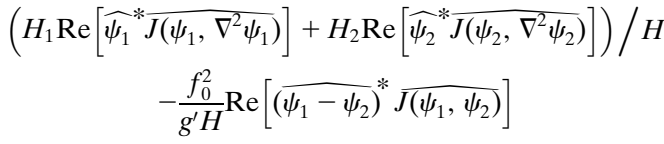 \\
\hline$\hat{D}$ & $-A_{h} \operatorname{Re}\left[H_{1}{\widehat{\psi_{1}}}^{*} \widehat{\nabla^{4} \psi_{1}}+H_{2}{\widehat{\psi_{2}}}^{*} \widehat{\nabla^{4} \psi_{2}}\right] / H$ \\
\hline
\end{tabular}

$$
\begin{aligned}
& \partial_{t} q_{1}+J\left(\psi_{1}, q_{1}\right)=A_{h} \nabla^{4} \psi_{1}, \\
& \partial_{t} q_{2}+J\left(\psi_{2}, q_{2}\right)=A_{h} \nabla^{4} \psi_{2},
\end{aligned}
$$

where the potential vorticity in each layer $q_{i}$ is given by

$$
\begin{aligned}
& q_{1}=\nabla^{2} \psi_{1}+\frac{f_{0}^{2}}{g^{\prime} H_{1}}\left(\psi_{2}-\psi_{1}\right), \\
& q_{2}=\nabla^{2} \psi_{2}+\frac{f_{0}^{2}}{g^{\prime} H_{2}}\left(\psi_{1}-\psi_{2}\right) .
\end{aligned}
$$

where $\psi$ is the streamfunction, $A_{h}$ is the horizontal biharmonic viscosity coefficient, $H_{1}$ and $H_{2}$ are the layer thicknesses, $f_{0}$ is the Coriolis parameter, $g^{\prime}$ is the reduced gravity, and $J(\cdot)$ is the Jacobian operator.

The equation for the depth averaged energy budget is obtained by multiplying Eqs. (A1) and (A2) by $\left(H_{1} / H\right) \psi_{1}$ and $\left(H_{2} / H\right) \psi_{2}$ respectively, where $H=H_{1}+$ $H_{2}$, and then adding them together. Applying the Fourier transform on the depth averaged energy equation yields Eq. (1), for which each term is detailed in Table A1.

\section{APPENDIX B}

\section{Dvar Parameterization}

Current mapping with the variational method (2Dvar) is performed by minimizing the following quadratic cost function (Yaremchuk and Sentchev 2009, 2011):

$$
\begin{aligned}
J= & \sum_{k=1}^{K}\left[\left(\hat{P}_{k} \mathbf{u}\right) \cdot \mathbf{r}_{k}-u_{k}\right]^{2}+\sum_{i, j}\left[W_{c}\left(\nabla^{2} \operatorname{curl} \mathbf{u}\right)_{i j}^{2}\right. \\
& \left.+W_{d}\left(\nabla^{2} \operatorname{divu}\right)_{i j}^{2}+W_{u}\left(\nabla^{2} \mathbf{u}\right)_{i j}^{2}\right],
\end{aligned}
$$

where $K$ is the number of observed radial currents $u_{k}$, $\hat{P}_{k} \mathbf{u}$ is the interpolation operator of the $2 \mathrm{D}$ velocity field $\mathbf{u}$ on the observation point $\mathbf{x}_{k}, \mathbf{r}_{k}$ is the unit vector pointing toward the radar, and $\left(W_{c}, W_{d}, W_{u}\right)$ are the weights used to enforce smoothness in the fields of curl, divergence and velocity, respectively. We parameterize these weights as

$$
W_{u}=\frac{K \nu^{2}}{2 N \delta x^{2}}, W_{c}=L_{c}^{2} W_{u}, W_{d}=L_{d}^{2} W_{u},
$$

where $\nu^{-2}$ is the signal-to-noise ratio, $N$ is the number of Cartesian grid points where the velocity field is mapped, $\delta x$ is the Cartesian grid spacing in both $x$ and $y$ directions, and $L_{c}$ and $L_{d}$ are typical wavelengths of the curl and divergence fields, respectively. These latter scales are estimated from a preliminary mapping of the velocity field using an optimal interpolation method (Kim et al. 2008). Our weights parameterization differs from that proposed by Yaremchuk and Sentchev (2009) because we scale the typical magnitude of $\nabla^{2}$ curlu as $U /$ $\left(L_{c} \delta x^{2}\right)$ instead of $U /\left(L_{c}^{2} \delta x\right)$, where $U$ is a typical magnitude of the velocity. The main consequence of this scaling is to allow for more small-scale structures to exist in the velocity field by significantly reducing the amplitude of the smoothing parameters.

\section{REFERENCES}

Arbic, B. K., and G. R. Flierl, 2003: Coherent vortices and kinetic energy ribbons in asymptotic quasi two-dimensional f-plane turbulence. Phys. Fluids, 15, 2177-2189, https://doi.org/10.1063/ 1.1582183 .

_ , K. L. Polzin, R. B. Scott, J. G. Richman, and J. F. Shriver, 2013: On eddy viscosity, energy cascades, and the horizontal resolution of gridded satellite altimeter products. J. Phys. Oceanogr., 43, 283-300, https://doi.org/10.1175/JPO-D-110240.1 .

—, M. Müller, J. G. Richman, J. F. Shriver, A. J. Morten, R. B. Scott, G. Sérazin, and T. Penduff, 2014: Geostrophic turbulence in the frequency-wavenumber domain: Eddy-driven low-frequency variability. J. Phys. Oceanogr., 44, 2050-2069, https://doi.org/10.1175/JPO-D-13-054.1.

Ardhuin, F., L. Marié, N. Rascle, P. Forget, and A. Roland, 2009: Observation and estimation of Lagrangian, Stokes, and Eulerian currents induced by wind and waves at the sea surface. J. Phys. Oceanogr., 39, 2820-2838, https://doi.org/ 10.1175/2009JPO4169.1.

Capet, X., P. Klein, B. L. Hua, G. Lapeyre, and J. C. McWilliams, 2008a: Surface kinetic energy transfer in surface quasigeostrophic flows. J. Fluid Mech., 604, 165-174, https:// doi.org/10.1017/S0022112008001110.

_ , J. C. McWilliams, M. J. Molemaker, and F. Shchepetkin, 2008b: Mesoscale to submesoscale transition in the California Current System. Part III: Energy balance and flux. J. Phys. Oceanogr., 38, 2256-2269, https://doi.org/10.1175/2008JPO3810.1.

Chapman, R. D., L. K. Shay, H. C. Graber, J. B. Edson, A. Karachintsev, C. L. Trump, and D. B. Ross, 1997: On the accuracy of HF radar surface current measurements: Intercomparisons with ship-based sensors. J. Geophys. Res., 102, 18 737-18748, https://doi.org/10.1029/97JC00049.

Charney, J. G., 1971: Geostrophic turbulence. J. Atmos. Sci., 28, 1087-1095, https://doi.org/10.1175/1520-0469(1971)028<1087: $\mathrm{GT}>2.0 . \mathrm{CO} ; 2$. 
Chavanne, C., P. Flament, and K. W. Gurgel, 2010: Interactions between a submesoscale anticyclonic vortex and a front. J. Phys. Oceanogr., 40, 1802-1818, https://doi.org/10.1175/2010JPO4055.1.

Emery, W. J., and R. E. Thomson, 2001: Data Analysis Methods in Physical Oceanography. Elsevier, 654 pp., https://doi.org/ 10.1016/B978-0-444-50756-3.X5000-X.

Forget, P., 2015: Noise properties of HF radar measurement of ocean surface currents. Radio Sci., 50, 764-777, https://doi.org/ 10.1002/2015RS005681.

Harris, F., 1978: On the use of windows for harmonic analysis with the discrete Fourier transform. Proc. IEEE, 66, 51-83, https:// doi.org/10.1109/PROC.1978.10837.

Held, I. M., R. T. Pierrehumbert, S. T. Garner, and K. L. Swanson, 1995: Surface quasi-geostrophic dynamics. J. Fluid Mech., 282, 1-20, https://doi.org/10.1017/S0022112095000012.

Kamli, E., C. Chavanne, and D. Dumont, 2016: Experimental assessment of the performance of high-frequency CODAR and WERA radars to measure ocean currents in partially icecovered waters. J. Atmos. Oceanic Technol., 33, 539-550, https://doi.org/10.1175/JTECH-D-15-0143.1.

Kim, S. Y., 2010: Observations of submesoscale eddies using highfrequency radar-derived kinematic and dynamic quantities. Cont. Shelf Res., 30, 1639-1655, https://doi.org/10.1016/j.csr.2010.06.011. , and P. M. Kosro, 2013: Observations of near-inertial surface currents off Oregon: Decorrelation time and length scales. J. Geophys. Res. Oceans, 118, 3723-3736, https://doi.org/ 10.1002/jgrc.20235.

— E. J. Terrill, and B. D. Cornuelle, 2008: Mapping surface currents from HF radar radial velocity measurements using optimal interpolation. J. Geophys. Res., 113, C10023, https:// doi.org/10.1029/2007JC004244.

Klein, P., B. L. Hua, G. Lapeyre, X. Capet, S. Le Gentil, and H. Sasaki, 2008: Upper ocean turbulence from high-resolution 3D simulations. J. Phys. Oceanogr., 38, 1748-1763, https:// doi.org/10.1175/2007JPO3773.1.

Lipa, B., and D. Barrick, 1983: Least-squares methods for the extraction of surface currents from CODAR crossed-loop data: Application at ARSLOE. IEEE J. Oceanic Eng., 8, 226-253, https://doi.org/10.1109/JOE.1983.1145578.

McWilliams, J. C., M. J. Molemaker, and I. Yavneh, 2001: From stirring to mixing of momentum: Cascades from balanced flows to dissipation in the oceanic interior. From Stirring to Mixing in a Stratified Ocean: Proc. 12th 'Aha Huliko'a Hawaiian Winter Workshop, Honolulu, HI, University of Hawai'i at Mānoa, 59-66, http://www.soest.hawaii.edu/PubServices/ 2001pdfs/McWilliams.pdf.

Mensa, J., M.-L. Timmermans, I. Kozlov, W. Williams, and T. Özgökmen, 2018: Surface drifter observations from the Arctic Ocean's Beaufort Sea: Evidence for submesoscale dynamics. J. Geophys. Res. Oceans, 123, 2635-2645, https:// doi.org/10.1002/2017JC013728.

Molemaker, M. J., J. C. McWilliams, and I. Yavneh, 2005: Baroclinic instability and loss of balance. J. Phys. Oceanogr., 35, 1505-1517, https://doi.org/10.1175/JPO2770.1.

,-- , and X. Capet, 2010: Balanced and unbalanced routes to dissipation in an equilibrated Eady flow. J. Fluid Mech., 654, 35-63, https://doi.org/10.1017/S0022112009993272.

Nadeau, L.-P., and D. N. Straub, 2009: Basin and channel contributions to a model Antarctic Circumpolar Current. J. Phys. Oceanogr., 39, 986-1002, https://doi.org/10.1175/2008JPO4023.1.

Nikurashin, M., G. K. Vallis, and A. Adcroft, 2013: Routes to energy dissipation for geostrophic flows in the Southern Ocean. Nat. Geosci., 6, 48-51, https://doi.org/10.1038/ngeo1657.
Ohlmann, C., P. White, L. Washburn, E. Terrill, B. Emery, and M. Otero, 2007: Interpretation of coastal HF radar-derived surface currents with high-resolution drifter data. J. Atmos. Oceanic Technol., 24, 666-680, https://doi.org/10.1175/JTECH1998.1.

Pawlowicz, R., B. Beardsley, and S. Lentz, 2002: Classical tidal harmonic analysis including error estimates in MATLAB using T_TIDE. Comput. Geosci., 28, 929-937, https://doi.org/ 10.1016/S0098-3004(02)00013-4.

Pearson, J., B. Fox-Kemper, R. Barkan, J. Choi, A. Bracco, and J. C. McWilliams, 2019: Impacts of convergence on structure functions from surface drifters in the Gulf of Mexico. J. Phys. Oceanogr., 49, 675-690, https://doi.org/10.1175/JPO-D-18-0029.1.

Poje, A. C., T. M. Özgökmen, D. J. Bogucki, and A. Kirwan, 2017: Evidence of a forward energy cascade and Kolmogorov selfsimilarity in submesoscale ocean surface drifter observations. Phys. Fluids, 29, 020701, https://doi.org/10.1063/1.4974331.

Salmon, R., 1980: Baroclinic instability and geostrophic turbulence. Geophys. Astrophys. Fluid Dyn., 15, 167-211, https:// doi.org/10.1080/03091928008241178.

_ 1998: Lectures on Geophysical Fluid Dynamics. Oxford University Press, $400 \mathrm{pp}$.

Scott, R. B., 2001: Evolution of energy and enstrophy containing scales in decaying, two-dimensional turbulence with friction. Phys. Fluids, 13, 2739-2742, https://doi.org/10.1063/1.1388181. - and F. Wang, 2005: Direct evidence of an oceanic inverse kinetic energy cascade from satellite altimetry. J. Phys Oceanogr., 35, 1650-1666, https://doi.org/10.1175/JPO2771.1.

— J. A. Goff, A. C. Naveira Garabato, and A. J. Nurser, 2011: Global rate and spectral characteristics of internal gravity wave generation by geostrophic flow over topography. J. Geophys. Res., 116, C09029, https://doi.org/10.1029/2011JC007005.

Soh, H. S., and S. Y. Kim, 2018: Diagnostic characteristics of submesoscale coastal surface currents. J. Geophys. Res. Ocean., 123, 1838-1859, https://doi.org/10.1002/2017JC013428.

Straub, D. N., and B. T. Nadiga, 2014: Energy fluxes in the quasigeostrophic double gyre problem. J. Phys. Oceanogr., 44, 1505-1522, https://doi.org/10.1175/JPO-D-13-0216.1.

Taylor, S., and D. Straub, 2016: Forced near-inertial motion and dissipation of low-frequency kinetic energy in a wind-driven channel flow. J. Phys. Oceanogr., 46, 79-93, https://doi.org/ 10.1175/JPO-D-15-0060.1.

Tulloch, R., J. Marshall, C. Hill, and K. S. Smith, 2011: Scales, growth rates, and spectral fluxes of baroclinic instability in the ocean. J. Phys. Oceanogr., 41, 1057-1076, https://doi.org/ 10.1175/2011JPO4404.1.

Vallis, G. K., 2006: Atmospheric and Oceanic Fluid Dynamics. Cambridge University Press, $745 \mathrm{pp}$.

Vanneste, J., 2013: Balance and spontaneous wave generation in geophysical flows. Annu. Rev. Fluid Mech., 45, 147-172, https://doi.org/10.1146/annurev-fluid-011212-140730.

_ , and I. Yavneh, 2004: Exponentially small inertia-gravity waves and the breakdown of quasigeostrophic balance. J. Atmos. Sci., 61, 211-223, https://doi.org/10.1175/15200469(2004)061<0211:ESIWAT > 2.0.CO;2.

Xie, J.-H., and J. Vanneste, 2015: A generalised-Lagrangian-mean model of the interactions between near-inertial waves and mean flow. J. Fluid Mech., 774, 143-169, https://doi.org/10.1017/jfm.2015.251.

Yaremchuk, M., and A. Sentchev, 2009: Mapping radar-derived sea surface currents with a variational method. Cont. Shelf Res., 29, 1711-1722, https://doi.org/10.1016/j.csr.2009.05.016.

_ mapping radar-derived sea surface currents. Cont. Shelf Res. 31, 758-768, https://doi.org/10.1016/j.csr.2011.01.009. 